\title{
The Electric Riverboat Charging Station Location Problem
}

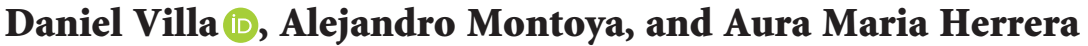 \\ Departamento de Ingeniería de Producción, Universidad EAFIT, Carrera 49 No. 7 Sur-50, Medellín, Colombia \\ Correspondence should be addressed to Daniel Villa; dvillab@eafit.edu.co
}

Received 27 November 2019; Revised 30 June 2020; Accepted 30 September 2020; Published 16 November 2020

Academic Editor: Maria C. Falvo

Copyright $\odot 2020$ Daniel Villa et al. This is an open access article distributed under the Creative Commons Attribution License, which permits unrestricted use, distribution, and reproduction in any medium, provided the original work is properly cited.

\begin{abstract}
Nowadays, the electric mobility is mainly focused on urban areas. However, the use of Photovoltaic-assisted Charging Stations (PVCSs) can contribute to implement the electric mobility in rural areas disconnected from the national grid. Inspired by the new river operations with an Electric Boat (EB), we introduce a new location problem named the Electric Riverboat Charging Station Location Problem (ERCSLP). This problem estimates the necessary infrastructure for an EB to be able to perform a round trip. In this case, we decide the location of the PVCSs and the size of the EB battery aiming to minimize the sum of the PVCS and the EB battery costs. In this problem, we include the nonlinear behavior of the charging function and the variation of the solar radiation. For solving this problem, we propose a Mixed-Integer Linear Programming (MILP) formulation. For testing this MILP formulation, we build a set of instances based on real river transport operations that have the potential to migrate to the electric mobility. In our computational experiments, we show that our MILP formulation can find the optimal solution of the instances. Finally, we perform a sensitivity analysis and an economic viability analysis of the electric mobility in these operations.
\end{abstract}

\section{Introduction}

Electric mobility is emerging as an attractive alternative to counteract the Greenhouse Gas (GHG) emissions and to reduce the dependency on fossil fuels. Different organizations from both the public and the private sectors have implemented the use of Electric Vehicles (EVs) for transport operations [1]. Most of these operations are found in urban areas, since the concentration of polluting gases is considerably higher compared to rural areas [2]. Additionally, the electric infrastructure is more developed in urban areas, which implies better energy availability, crucial for EVs' driving range limitations [1]. However, this does not mean that the development of the electric mobility is not important in rural areas. The use of fossil fuels in rural areas still accounts for $\mathrm{CO}_{2}$ emission and can potentially contaminate natural resources such as water bodies. But, the implementation of transport operations with EVs in rural areas has been limited by the long distances that are normally greater than the driving range of the EVs. Besides, the grid infrastructure is often unreliable or it is even nonexistent.

Nevertheless, recent technological developments aim to overcome the limitations of the EVs for transport operations in rural areas. Some of the most important technological improvements are addressed to both Battery Energy Density (BED) and Photovoltaic-assisted Charging Stations (PVCSs). The BED is the quantity of energy that a battery is capable to store per unit of mass $(\mathrm{kWh} / \mathrm{kg})$. Over the last few years, the BED has substantially increased through the improvement of the materials for both anode and cathode [3]. This improvement of the BED is fundamental for rural transport operations, as it increases the driving range of EVs. This allows them to perform routes that were not possible before. Furthermore, the PVCSs are the charging system that use solar panels for generating electric power, and some of them have a battery to store energy. Understanding that the rural areas are poorly connected to the national grid, the use of PVCSs rises as a good alternative for supporting the electric mobility in these areas. To help with the implementation of this technology, manufacturers have lowered the manufacturing cost, thanks to different improvements in conversion technologies and installation processes $[4,5]$.

In rural areas, there are different types of transport operations that can be developed around electric mobility systems. One of these operations is the river transport for both passengers and freight in areas with no connection to 
the national grid, for instance, the river transport operation in the Amazon region. In this region, approximately $90 \%$ of the transport operations are carried out by river, due to its lack of road connectivity $[6,7]$. A problem of river transport operations with Internal Combustion Boats (ICBs) is related to the unreliable and inconstant supply of fuel. This factor could also affect the quality of life of the inhabitants of these isolated areas. Another problem of using ICBs is linked to its impact on the water sources due to the increase of noise and fuel spillage. This can represent a threat to the species that dwell in those ecosystems, which have suffered dramatic reductions in their populations during the last few years [8]. As can be seen, Electric Boats (EBs) have several advantages compared to ICBs as the reduction of $\mathrm{CO}_{2}$ emissions and the reduction of noise affecting the fauna [9]. For these reasons, in some places of the Amazon basin, people are starting to use EBs as an alternative to ICBs. Some examples of river transport operations with EBs have been introduced by several authors. Jaimurzina et al. [7] presented a solution for the Putumayo River in Ecuador; Ponce [9] presented a study for the insertion of an electrosolar boat in the Napo River in Ecuador; and Miniguano and Elizabeth [10] described the Kara project, which intends to develop a transport system for native communities in two Ecuadorian rivers.

Although EBs are an excellent alternative to perform river transport operations, it must be considered that the communities that inhabit these zones, near from the river, are isolated from each other. Therefore, the EBs would need to move across large distances, which occasionally could be larger than the autonomy of the EBs. For avoiding this problem, a possible solution would be the installation of a higher battery capacity; however, this solution requires a higher investment while reducing the efficiency of the EBs. The latter happens due to the energy consumption being directly related to the weight of the battery. A different solution for improving the autonomy would be the installations of Charging Stations (CSs) along the river. Nevertheless, it is important to be aware that this remote area is not connected into the national power grid. For example, in Colombia, $51 \%$ of the territory is not connected to the grid. Most of this area is located in the Amazon region, which represents $41.8 \%$ of the national territory [11]. For addressing this problem, PVCSs arise as a promising solution, as this type of CS does not need to be connected to the grid. Prior to implementation of this solution, there are two questions to be answered. Firstly, where should the PVCSs be located in such a way that the EB is capable of completing its route? For the second question, it is important to consider two possible scenarios. In the first scenario, the EB has enough battery capacity to complete the route (without using any intermediate PVCSs). In the second scenario, the EB has a smaller battery capacity and uses intermediate PVCSs. With this in mind, the second question is which scenario is more cost effective?

For answering these questions, it is necessary to consider an optimization problem that allows determining the capacity of the EB battery and the location of the PVCSs minimizing the total investment cost, defined as the PVCS cost plus the EB battery cost. In this optimization problem, it is important to consider several components of the electric mobility system, such as the solar radiation and the energy generation of the PV system, nonlinear charging functions for the Li-ion batteries, and energy consumption estimations. Additionally, it is worth noting that there is a trade-off between the investment in the capacity of the EB battery and the amount of PVCSs to place. In this paper, we formally define this problem as the Electric Riverboat Charging Station Location Problem (ERCSLP).

For solving this problem, we propose a Mixed-Integer Linear Programming (MILP) formulation. To test the performance of the MILP formulation, we used a set of instances built from ICB real river operations in Colombia that have the potential to operate with EBs. The results show that the MILP formulation is capable of solving the test instances in competitive CPU times. The results also show that, for some instances, even if it is possible to complete the route with a big battery without PVCSs, the optimal solution contemplates the installation of PVCSs while using small batteries. Additionally, we compare the investment costs of the electric mobility system against the fuel costs of the operation with an ICB, for a time horizon of 10 years (considering the battery lifespan). For several instances, it is possible to recover the investment of the electric mobility system in 10 years or less.

The remainder of this paper is organized as follows. Section 2 reviews the literature related to the problem. Section 3 briefly explains some technical issues about electric mobility, aiming to understand the formulation of the problem. Section 4 formally introduces the ERCSLP and presents a MILP formulation for solving the problem. Section 5 presents the computational experiments performed for the ERCSLP. Finally, Section 6 concludes the paper. For easy reference, Appendix A summarizes our notation.

\section{Literature Review}

In the last few years, the CS location problem literature has been gaining importance; however, most researchers have focused their attention on the urban context and applications where the CSs are connected to the grid. CS location problems have been addressed from two different perspectives: one from the CS user and the other from the CS owner. The former is related to optimizing objective functions that impact the user; for example, minimizing the traveled distance [12], reducing the total number of recharges $[13,14]$, and reducing the travel time [15]. On the other hand, the owner's perspective is related to optimizing objective functions that impact the interest of the CSs' owner, for example, minimizing the installation costs [16], maximizing the coverage $[17,18]$, minimizing new CSs deployment costs [19], and maximizing the number of EVs served [20].

Recently, some researchers have integrated Renewable Energy Sources (RESs) to CS location problems. In this way, Moradi et al. [21] presented a model for locating and sizing CSs with RESs, where the CSs work on grid connected systems because their objectives are to reduce grid power 
losses, improve voltage stability, and minimize EV charging costs. In a different study, Mozafar et al. [22] designed a model that aims to the optimal location of RESs and CSs. Their objective function is to minimize a multiobjective function composed by voltage fluctuations, energy supplying costs, and power losses. Similarly, Amini et al. [23] proposed a model for the simultaneous location of EVs parking lots and distributed renewable resources in a smart power distribution network. Their approach has two phases. In the first phase, they simultaneously determined candidate locations for both EV parking lots and RES. In the second phase, they considered the distribution of RES and their penetration.

The problem that we address in this paper can be seen in two levels: strategic and operative. On the strategic level, we determine the CS location and the EB battery capacity. On the operative level, we consider operational decisions such as where and how much to charge accounting for operative constraints. In the literature, we have found other related type of problems that also consider two levels on the decision-making process, known as location-routing problems with EVs. These problems consider the decisions involved in the CS location (strategic level) and the routing of the EVs (operative level). In this context, the studies in the literature consider the autonomy restrictions of the EVs and their charging times to complete their operations. The locationrouting problems with EVs can be traced back to Yang and Sun [24], where they presented a model to determine battery swap station locations and, simultaneously, design a routing plan for a fleet of EVs for the delivering of goods. In this problem, the authors considered the EV driving range limitations and different demands from the customers that had to be satisfied. Li-ying and Yuan-bin [25] presented an extension of the work by Yang and Sun [24], in which they made decisions about CS infrastructure and their location. Charging infrastructure is referred to the technology of the $\mathrm{CS}$, which can impact the charging times. Additionally, they decided the vehicle-routing plan considering Time Windows (TWs) for a set of customers. Schiffer and Walther [26] proposed a location-routing problem with TWs and partial recharging. Recently, Schiffer and Walther [27] presented an extension on their work, including the acquisition of the EVs. Another approach is presented by Cui et al. [28] where they present a location-routing problem; they present a location-routing problem for Mobile Charging Vehicles (MCVs) considering fixed candidate locations and, after locating the CSs and determining their charging rates, routing the MCVs to the EVs that are requiring the service.

Considering that ERCSLP is in the context of a river operation, the EB has to state on a fixed route. To the best of our knowledge, there is no previous work on CS location in a fixed route context. Nonetheless, in the literature, there are works related to the charging decision problem for EVs in a fixed route. This problem was introduced by Montoya et al. [29]; in this study, they presented this problem as a subproblem of an electric vehicle-routing problem with nonlinear charging functions. To solve this problem, they proposed a MILP formulation and a heuristic approach. Recently, Villa et al. [30] introduced a problem of a fixed route for a river transport operation with EBs considering speed and charging decisions for minimizing charging costs and battery degradation costs.

The previous studies on CS location have addressed the urban context and land applications, while rural areas have received scarce attention. Moreover, nobody has studied the CS location problem for a river transport operation, considering PV generation in standalone applications. In this regards, the closest work has been proposed by Zhang et al. [31]. This work presents a CSs location problem for Electric Ships (ESs). However, they did not consider fixed routes as their context is on a lake. Their problem is divided into three phases: finding the candidate sites to be CSs, selecting the CSs among the candidates, and finally, determining the capacity of the CSs. They present a MILP formulation for solving this problem.

\section{Technical Issues about the ERCSLP}

In the problem that we are facing in this study, we include different components related to electric mobility such as energy consumption models, PV generation, and nonlinear charging times. Aiming to give the reader a better understanding of these components, we present a short explanation for each one.

3.1. Energy Consumption Models. When planning a transport operation system, in the context of the electric mobility, it is necessary to estimate the energy consumption of the EV, or in this case the EB. For the case of EVs, Villa and Montoya [32] made taxonomy of some energy consumption models in the literature. In this study, they found 25 models to estimate the energy consumption of the EVs. However, in the case of the EBs, the number of models to estimate the energy consumption is more limited. Minami and Yamachika [33] pioneered the study of the models that estimate the energy consumption of the EBs. In their work, they reviewed some previous models and proposed a new model. Additionally, they compared the accuracy of the model using experimental data. In our case, the energy consumption is an input parameter; therefore, any model could be used for the estimating the consumption. However, it is important to note that there are some key features for the estimation such as EB and river speed, EB dimensions, and EB weight. The latter is important, because the EB weight affects the power needed to propel the EB. The weight of the EB itself depends on its feature and the EB size. Considering that the battery size is a decision variable for this problem, the energy consumption parameter will be in terms of the EB battery size. Although, in our model, the energy consumption is an input parameter, it is important to mention that, in the case of restrictive waters (e.g., the rivers), the propulsive power of a boat is sensitive to the width, depth, and the current of the river. One of the first authors to analyze the impact of the depth on the propulsion quality was Schwanecke [34]. This author proposed a model for analyzing the hydrodynamic and the propulsion of a boat on restricted water depth. Recently, Thi Ngoc Hoa et al. [35] investigated the impact of the 
depth of the waterway on the ship's resistance and propulsion using a computational fluid dynamic method.

3.2. Nonlinear Charging Function. The battery charging function is nonlinear, which seeks to avoid overcharging degradation. This function represents the relationship between the energy level of the battery and its charging time. The charging process is represented in a Constant Current (CC)-Constant Voltage (CV) scheme. Initially, a CC phase is performed, in which the current is held constant while the battery's terminal voltage is below a maximum value. Then, the CV phase begins. In this phase, the current starts to decrease to hold the terminal voltage constant. This process causes a change in the linearity of the charging time [36]. Montoya et al. [29] used a piecewise linear approximation to represent the nonlinear charging function. Their approximation obtains an average relative absolute error of less than $2 \%$ for the data that they used in their experiments. Figure 1 shows a representation of a piecewise linear approximation. The data were obtained from a battery capacity of $16 \mathrm{kWh}$, using a charger of $22 \mathrm{~kW}$ of power. The breakpoints are represented in the coordinates shown in Figure 1. Now, the values of the breaking points depend on the size of the battery and the charging power supplied.

\subsection{Photovoltaic-Assisted Charging Stations Behavior. As} mentioned above, the increasing interest on the implementation of green renewable energies has impacted the development of energies related to CS systems. These developments have focused not only on the energy capture stages but also on the storage, tracking systems, and energy conversion. All these elements have a direct or indirect impact on the generation capacity of the PVCS. In this section, we intend to give a brief and simple description of a PVCS operation considering the relevant factors that affect the strategic decisions of this problem. For further description of the topics mentioned here, refer to [37] and [38].

A PVCS consists of several components: a set of photovoltaic modules converting solar radiation into electric power; a DC-DC power converter with an algorithm that optimizes the power curve of the PV module; an internal battery for energy storage, called the Energy Storage System (ESS); and a DC charger that controls the input current to the EB. Figure 2 depicts a general scheme for a standalone PVCS with a battery connected from an EV. Some standalone PVCSs could work without an ESS; however, this is impractical as the incoming radiation fluctuates; and thus, the generation is insufficient for charging the EB in short times [38]. Our study considers standalone PVCSs with an ESS.

For the PVCSs, the input power may vary depending on environmental factors such as temperature and geographical position of the photovoltaic modules. Considering this variation of the input energy, we present three possible scenarios for the PVCS operation. In the first scenario, we have a PVCS without any EB connected to it. In this scenario, the energy generated by the PV module flows directly to the ESS until it is fully charged. With standalone PVCSs, once the ESS capacity is full, the additional generated energy is lost. For the remaining two scenarios, we will consider an energy lower bound for the ESS, to avoid degradation due to the overdischarging. With this in mind, for the second scenario, the energy within the ESS is above the lower bound, and an EB is connected to the PVCS. In this case, the energy for charging the EB comes from the PV modules and simultaneously from the ESS, in order to ensure the charging power selected to charge the EB. It is important to mention that, in this scenario, the charging time is determined by the nonlinear charging function. Finally, in the last scenario, the energy within the ESS is below the lower bound, and an EB is connected to the PVCS. For this scenario, we consider that there is a control system that ensures that the energy for charging the EB comes only from the PV modules. In this case, the charging time will only depend on the PV generation.

For the simplification of the modeling, we consider that PV modules are always in a horizontal position (the PVCS does not have a tracking system). Additionally, solar radiation is discretized in a finite number of time intervals, and the panel efficiency is considered constant for the whole day. Figure 3 shows the average solar radiation in $\left(\mathrm{W} / \mathrm{m}^{2}\right)$ per hour, in the month of June, for the municipality of Sincelejo, in Colombia.

\section{Problem Description and Formulation}

4.1. Problem Description. Let $N=\{0, \ldots, r, \ldots, n\}$ be a set of nodes that compose a round trip on a river (i.e., the EB should visit these nodes). The nodes 0 and $n$ represent the same node, where 0 is the starting point and $n$ is the final destination. The node $r$ represents the return node. The subsets $N_{o}=\{1, \ldots, r-1\}$ and $N_{r}=\{r+1, \ldots, n-1\}$ represent the nodes to visit on the outbound and return trip. Considering that we assume that the EB visits the same nodes on the on the outbound and return trip, for this problem, the nodes in $N_{r}$ are a copy of the nodes in $N_{o}$. For instance, the node $n-1$ is a copy of node 1 , and the node $r+1$ is a copy of $r-1$. Therefore, a PVCS located at node $i \in N_{o}$ could serve to charge the EB also on the return trip. It is important to note that if node $j \in N_{r}$ is a copy of node $i \in N_{o}$, their service times and TWs could be different. Each node $i \in N \backslash\{0, n\}$ has a service time $o_{i}$ (e.g., load and unload times, lunch breaks, and sleep times). Each node $i \in N \backslash\{0\}$ has a TW $\left[s_{i}, l_{i}\right]$, where $s_{i}$ and $l_{i}$ are the earliest and latest possible arrival times. The travel time between nodes $i-1$ and $i \in N \backslash\{0\}$ is defined as $t_{i}$. The EB must return to the final destination $n$ before a time limit $T_{\max }$. Let $M=\left\{Q_{\min }, \ldots, Q_{\max }\right\}$ be a set of battery capacities for the EB. Each battery capacity $m \in M$ has associated an acquisition cost $c_{m}$ (in $\$$ ). It is assumed that the EB leaves the origin with a fully charged battery. The energy consumption between nodes $i-1$ and $i \in N \backslash\{0\}$ using a EB with a battery capacity of $m \in M$ is defined as $e_{i m}$. Let $H$ be a set of different types of PVCSs' technologies. Each technology is related with the charging rate (e.g., slow, moderate, and fast). The cost of installing a PVCS at the node $i \in N \backslash\{0, n\}$ of type $h \in H$ is defined as 


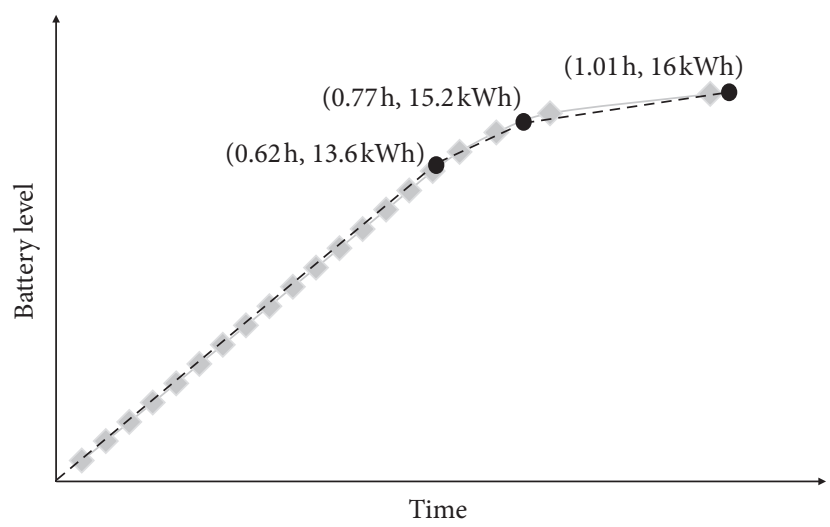

FIgURE 1: Piecewise linear approximation for charging a battery of $16 \mathrm{kWh}$ with a charging rate of $22 \mathrm{~kW}$. Source: [30].

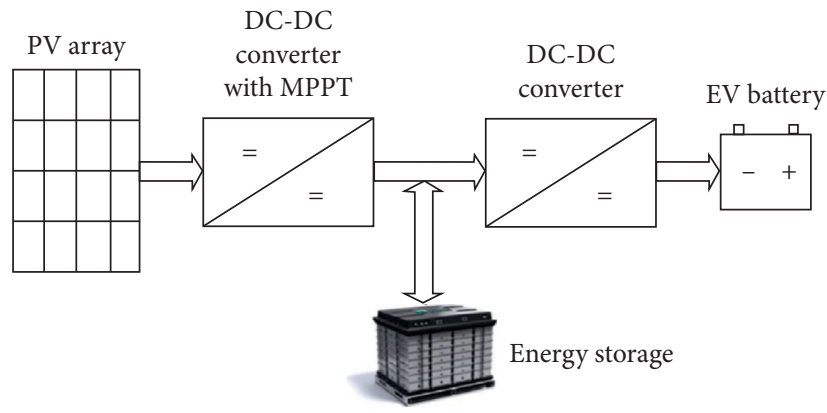

Figure 2: General scheme of a PVCS. Source: [38].

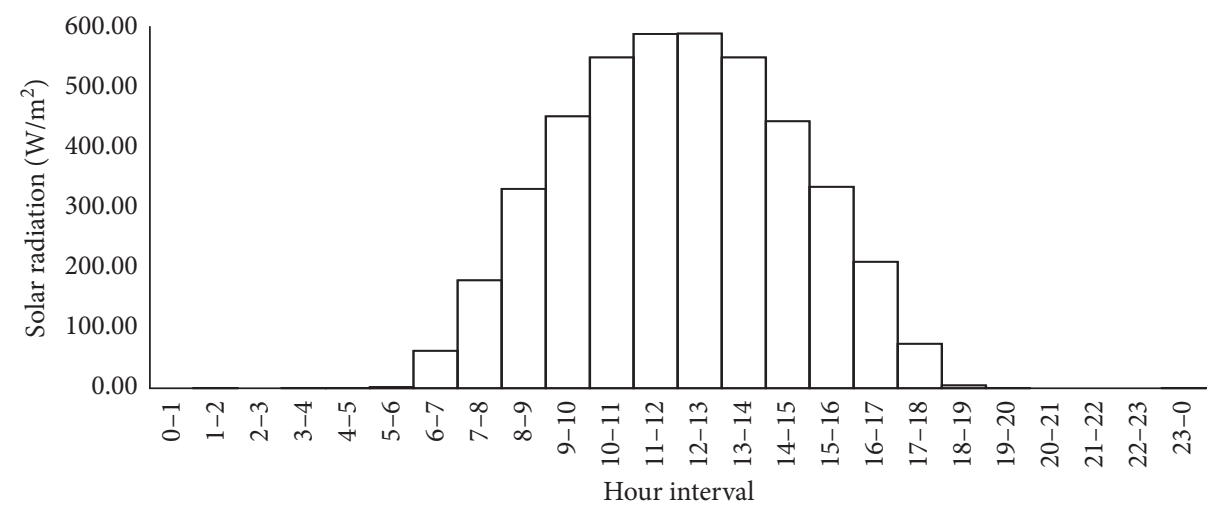

FIgURE 3: Solar radiation curve for the municipality of Sincelejo, Colombia. Source: data from IDEAM [39].

$f_{i h}$. For modeling the nonlinear charging behavior, we use a piecewise linear approximation with a set of break points $B=\{0,1, \ldots, b\}$. Each segment of the piecewise linear between breakpoints $k-1$ and $k \in B \backslash\{0\}$ has a slope $\rho_{k h}$ according to a charging rate $h \in H$, and each segment is bounded by the battery levels $\alpha_{k-1, m h}$ and $\alpha_{k m h}$ for a battery capacity of $m \in M$. For modeling the PV generation, let $T=\left\{1,2, \ldots, T_{\max }\right\}$ be the set of time intervals and $\Delta$ be the length of each interval. Each time interval $j \in T$ has an upper bound $u_{j}$ (in hours) and a power generation $g_{j}$ (in $\mathrm{kW}$ ). The latter is considered constant during each time interval. Finally, the PVCSs have an ESS capacity of size $a$ (in $\mathrm{kWh}$ ) and a lower energy limit $v$ (in $\mathrm{kWh}$ ) for preventing the overdischarge of the ESS.
In the ERCSLP, the objective is to find the EB's battery capacity and the number of located PVCSs such that the total cost of the system is minimized. This problem considers TWs and operation times for the EB at each node, the nonlinear behavior of the charging function, the variation of the energy generation of the PVCSs, the autonomy of the EB (defined by the battery capacity), and the time limit of the route.

4.2. Illustrative Example. In this section, we present a numerical example intending to give the reader a better perspective of our problem. Figure 4 shows a numerical example illustrating the ERCSLP. In Figure 4(a), we depict a 


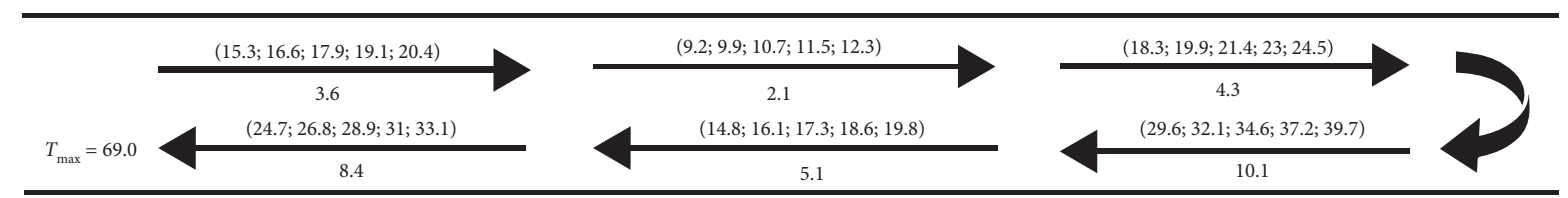

\begin{tabular}{|l|c|}
\hline \multicolumn{1}{|c|}{$\mid 0$} \\
\hline \multicolumn{2}{|c|}{ Location cost (\$) } \\
\hline Slow; fast) & - \\
\hline \multicolumn{2}{|c|}{ Time window (h) } \\
\hline Outbound & - \\
\hline Return & {$[68.4 ; 69.0]$} \\
\hline \multicolumn{2}{|c|}{ Service time (h) } \\
\hline Outbound, return) & $(-;-)$ \\
\hline
\end{tabular}

(1)

\begin{tabular}{|l|c|}
\hline \multicolumn{2}{|c|}{ Location cost $(\$)$} \\
\hline (Slow; fast) & $(25,193 ; 26,793)$ \\
\hline \multicolumn{2}{|c|}{ Time window (h) } \\
\hline Outbound & {$[4.6 ; 5.6]$} \\
\hline Return & {$[56.2 ; 57.2]$} \\
\hline \multicolumn{2}{|c|}{ Service time (h) } \\
\hline (Outbound, return) & $(2.0 ; 0.75)$ \\
\hline
\end{tabular}

Origin/destination

A Return node

0

Intermediate node

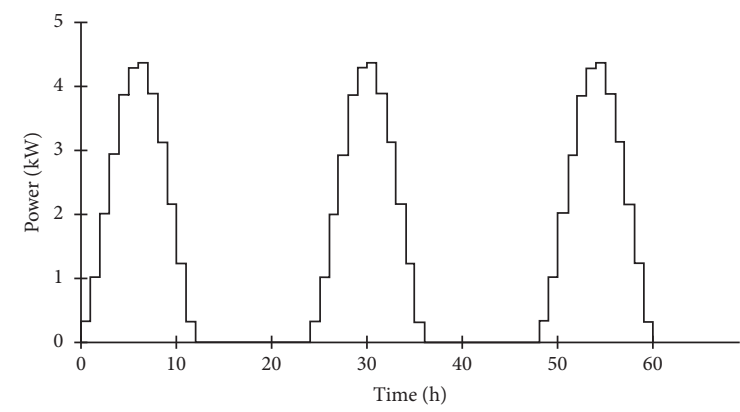

(b)

\begin{tabular}{|c|c|}
\hline \multicolumn{2}{|c|}{ Location cost (\$) } \\
\hline (Slow; fast) & $(25,373 ; 26,973)$ \\
\hline \multicolumn{2}{|c|}{ Time window $(\mathrm{h})$} \\
\hline Outbound & {$[9.5 ; 11.5]$} \\
\hline Return & {$[39.2 ; 41.2]$} \\
\hline \multicolumn{2}{|c|}{ Service time (h) } \\
\hline (Outbound, return) & $(10.0 ; 10.0)$ \\
\hline
\end{tabular}
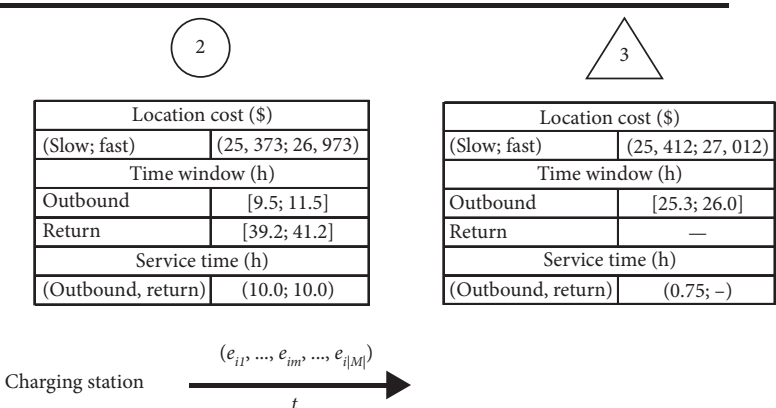

(a)

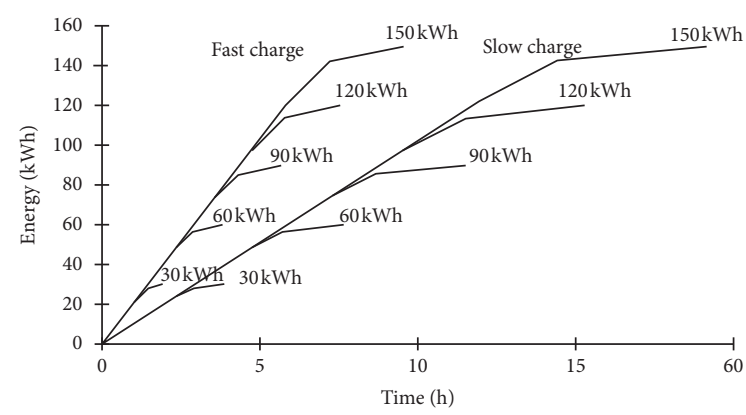

(c)
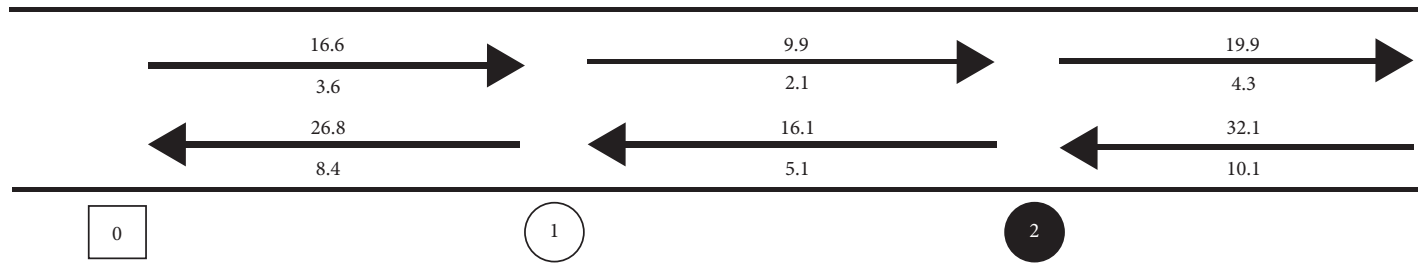

\begin{tabular}{|l|c|c|}
\cline { 2 - 3 } \multicolumn{1}{c|}{} & Outbound & Return \\
\hline Arrival time & - & 68.9 \\
\hline Departure time & 0.0 & - \\
\hline $\begin{array}{l}\text { Energy level } \\
\text { arrival }\end{array}$ & - & 0.0 \\
\hline $\begin{array}{l}\text { Energy level } \\
\text { departure }\end{array}$ & 60.0 & - \\
\hline
\end{tabular}

\begin{tabular}{|l|c|c|}
\cline { 2 - 3 } \multicolumn{1}{c|}{} & Outbound & Return \\
\hline Arrival time & 5.6 & 57.0 \\
\hline Departure time & 8.9 & 60.5 \\
\hline $\begin{array}{l}\text { Energy level } \\
\text { arrival }\end{array}$ & 43.4 & 26.8 \\
\hline $\begin{array}{l}\text { Energy level } \\
\text { departure }\end{array}$ & 43.4 & 26.8 \\
\hline
\end{tabular}

\begin{tabular}{|l|c|c|}
\cline { 2 - 3 } \multicolumn{1}{c|}{} & Outbound & Return \\
\hline Arrival time & 11.0 & 41.0 \\
\hline Departure time & 21.0 & 52.0 \\
\hline $\begin{array}{l}\text { Energy level } \\
\text { arrival }\end{array}$ & 33.5 & 0.0 \\
\hline $\begin{array}{l}\text { Energy level } \\
\text { departure }\end{array}$ & 52.0 & 42.8 \\
\hline Charged energy & 18.5 & 42.8 \\
\hline $\begin{array}{l}\text { ESS Energy } \\
\text { level arrival }\end{array}$ & 41.3 & 52.7 \\
\hline $\begin{array}{l}\text { ESS Energy } \\
\text { level departure }\end{array}$ & 23.1 & 16.2 \\
\hline
\end{tabular}

(d)

FIGURE 4: Fixed route for an EB in a river transport operation. (a) Instance information. (b) Power generated in terms of solar radiation. (c) Piecewise linear approximation. (d) Instance solution.

round trip route where the EB departs from an origin node (0), which will also be the final destination of the route; two intermediate nodes; and a return node where the EB arrives and, afterwards, starts the return trip. This means that the EB sequentially visits nodes 1,2 , and 3 . Once it completes the outbound trip, it starts with the return trip visiting, once more, nodes 2 and 1 finally arriving to node 0 .

Each pair of nodes has associated a travel time (in hours) and energy consumption (in $\mathrm{kWh}$ ) for both the outbound and the return trips. Considering that the weight of the EB battery affects the energy consumption, for each pair of nodes, we have an energy consumption value associated to each possible battery size. For this example, the battery capacity is discretized in modules of $30 \mathrm{kWh}$ each, i.e., $(30,60, \ldots, 150 \mathrm{kWh})$, and the cost of each module will be 9,000(300. In Figure 4(a), above the arrows, we show the energy consumption for each possible battery capacity, and at the bottom, we show the travel time. Each node in the route has the costs for opening different types of PVCSs (slow or fast); its time window; and its service time. Each 
PVCS that is candidate to be opened has a battery capacity of $60 \mathrm{kWh}$ and an energy lower bound 20\% of its capacity. For this example, the maximum trip duration $\left(T_{\max }\right)$ is $69 \mathrm{~h}$. Assuming that the trip starts at sunrise, Figure 4(b) shows the solar value radiation per hour until $T_{\max }$. Figure 4 (c) presents the piecewise linear charging function for each of the battery capacities, as well as the two considered charging technologies (slow and fast).

Figure $4(\mathrm{~d})$ presents a feasible solution for this instance. In this solution, the selected EB battery capacity is $60 \mathrm{kWh}$. Each node has associated a table that shows both time and energy levels when the EB arrives to and departs from the node (for both the outbound and the return trips). One PVCS of slow charging technology is opened at node 2. For this node in particular, the table also shows the charged energy by the EB and the energy level of the ESS when the EB arrives to and departs from the PVCS. The objective function for this feasible solution is $\$ 43,373$; this result comprises the PVCS' cost and the EB battery's cost. For the sake of comparison, another feasible solution considers an EB with a battery capacity of $150 \mathrm{kWh}$, and no PVCS is opened. However, in this solution, the objective function is $\$ 45,000$, which is higher than the solution with a lower EB battery capacity and a PVCS opened.

Considering that the PVCS is opened at node 2, we will only detail how the system behaves at this node. As we mentioned above, when the EB departs from the origin, the energy level of the ESS corresponds to the lower bound, for example, $12 \mathrm{kWh}$. For this example, the EB departs from the origin at $2.1 \mathrm{~h}$, and it remains $3.2 \mathrm{~h}$ at node 1 . Then, the EB arrives at node 2 after $11 \mathrm{~h}(2.1+3.6+3.2+2.1)$, with an energy level of $33.5 \mathrm{kWh}$. For this $11 \mathrm{~h}$, the PV modules have generated $29.3 \mathrm{kWh}$. This energy has flowed into the ESS reaching a level of $41.3 \mathrm{kWh}$. The charging power of the PVCS is $11 \mathrm{~kW}$. Considering that the EB needs $18.5 \mathrm{kWh}$, the charging time will be $1.8 \mathrm{~h}$ using this charging power. For computing the charging time, we use the piecewise linear charging function with a charging power of $11 \mathrm{~kW}$ and the selected battery of $60 \mathrm{kWh}$. During the charging time, the PV modules generated $0.3 \mathrm{kWh}$. The battery level of the ESS when the EB finishes its charging process is $23.1 \mathrm{kWh}$. This is computed as the ESS battery level when the EB arrives at PVCS (41.3 kWh) plus the generated energy during the charging time $(0.3 \mathrm{kWh})$ minus the charged energy by the EB $(18.5 \mathrm{kWh})$. In the return trip, when the EB arrives at node 2 , after visiting node 3 , its energy level is $0 \mathrm{kWh}$. At this moment, the available energy at the ESS is $52.7 \mathrm{kWh}$ (it has generated an additional $29.6 \mathrm{kWh}$ since the departure). At this point, the EB requires to charge $42.8 \mathrm{kWh}$, for completing the route. The charging process begins at $41 \mathrm{~h}$, which is night time; Figure 4(b). Under this condition, the EB only takes energy from the ESS until it reaches the lower bound. At that moment, the EB has not completed its required charge (missing $2.1 \mathrm{kWh}$ ). This first charging process ends at $44.8 \mathrm{~h}$ (still night time). Therefore, the EB needs to wait that the PV modules resume generation of the remaining $2.1 \mathrm{kWh}$. The PV modules start the generation at $48 \mathrm{~h}$, and the $2.1 \mathrm{kWh}$ are generated at $50.4 \mathrm{~h}$. At this moment, the EB is ready to depart and complete the route.
4.3. Mixed-Integer Linear Programming Formulation. We now provide a MILP formulation for the ERCSLP. The MILP formulation uses the following decision variables:

(i) $w_{m}: 1$ if the EB battery capacity takes a value of $m \in M$, and 0 otherwise

(ii) $y_{i h}: 1$ if a PVCS of type $h \in H$ is opened at node $i \in N \backslash\{0, n\}$, and 0 otherwise

(iii) $x_{i}$ : battery level when the EB arrives at node $i \in N \backslash\{0\}$

(iv) $\tau_{i}$ : arrival time of the EB at node $i \in N \backslash\{0\}$

(v) $\Omega_{i k h}$ : charged energy when the EB charges at the PVCS of type $h \in H$ located at node $i \in N \backslash\{0, n\}$ between breakpoints $k-1$ and $k \in B \backslash\{0\}$

(vi) $\mu_{i k h}$ : battery level when the EB charges at the PVCS of type $h \in H$ located at node $i \in N \backslash\{0, n\}$ between breakpoints $k-1$ and $k \in B \backslash\{0\}$

(vii) $\theta_{i k m h}: 1$ if the EB charges on the segment between breakpoint $k-1$ and $k \in B \backslash\{0\}$ using a battery capacity of $m \in M$ at the node $i \in N \backslash\{0, n\}$, and 0 otherwise

(viii) $\phi_{i}$ : charging time at the PVCS located at node $i \in N \backslash\{0, n\}$

(ix) $\delta_{i}$ : stay time at the PVCS located at node $i \in N \backslash\{0, n\}$

(x) $\gamma_{i j}$ : Fraction of the time interval $j \in T$ that is being used to generate energy before the arrival of EB at the PVCS located at node $i \in N \backslash\{0, n\}$

(xi) $p_{i j}$ : fraction of the time interval $j \in T$ that is being used to generate energy during the charging process of EB at the PVCS located at node $i \in N \backslash\{0, n\}$

(xii) $z_{i j}: 1$ if the PVCS located at node $i \in N \backslash\{0, n\}$ is generating energy before the arrival of the $\mathrm{EB}$ at the time interval $j \in T$

(xiii) $d_{i j}: 1$ if the PVCS located at node $i \in N \backslash\{0, n\}$ is generating energy during the charging process of the EB at the time interval $j \in T$

(xiv) $\lambda_{i}$ : total energy generated by the PVCS located at node $i \in N \backslash\{0, n\}$ before the arrival time of the EB

(xv) $\psi_{i}$ : available energy at the PVCS located at node $i \in N \backslash\{0, n\}$ before the arrival time of the EB

(xvi) $\varphi_{i}$ : energy generated while the EB is charging at the PVCS located at node $i \in N \backslash\{0, n\}$

The MILP formulation of the ERCSLP is as follows:

$$
\min \sum_{m \in M} c_{m} w_{m}+\sum_{i \in N \backslash\{0, n\}} \sum_{h \in H} f_{i h} y_{i h}
$$

The objective function (1) seeks to minimize the total cost of the electric mobility system, which is composed of the PVCSs' installation cost and the cost of the EB battery.

Subject to 


$$
\sum_{m \in M} w_{m}=1
$$

Constraint (2) ensures that exactly one battery capacity is selected for the EB.

$$
\begin{aligned}
& x_{1}=\sum_{m \in M} m w_{m}-\sum_{m \in M} e_{1 m} w_{m}, \\
& x_{i}=x_{i-1}+\sum_{k \in B \backslash\{0\}} \sum_{h \in H} \Omega_{i-1, \mathrm{kh}}-\sum_{m \in M} e_{i m} w_{m}, \quad \forall i \in N \backslash\{0,1\},
\end{aligned}
$$$$
x_{i}+\sum_{k \in B \backslash\{0\}} \sum_{h \in H} \Omega_{i k h} \leq \sum_{m \in M} m w_{m}, \quad \forall i \in N \backslash\{0, n\} .
$$

Constraints (3) and (4) track the energy level when the EB arrives to node $i \in N \backslash\{0\}$ considering the charged energy at the located PVCSs. Constraint (5) ensures that the energy level of the EB does not exceed the maximum selected battery capacity.

$\mu_{i 1 h}=x_{i}+\Omega_{i 1 h}, \quad \forall i \in N \backslash\{0, n\}, \forall h \in H$,

$\mu_{i k h}=\mu_{i, k-1, h}+\Omega_{i k h}, \quad \forall i \in N \backslash\{0, n\}, \forall k \in B \backslash\{0,1\}, \forall h \in H$,

$\mu_{i k h} \geq \sum_{m \in M} \alpha_{k-1, m h} \theta_{i k m h}, \quad \forall i \in N \backslash\{0, n\}, \forall k \in B \backslash\{0,1\}, \forall h \in H$,

$$
\begin{aligned}
& \mu_{i k h} \leq \sum_{m \in M} \alpha_{k m h} \theta_{i k m h}+\left(1-\sum_{m \in M} \theta_{i k m h}\right) Q_{\max }, \\
& \quad \forall i \in N \backslash\{0, n\}, \forall k \in B \backslash\{0\}, \forall h \in H,
\end{aligned}
$$

$\mu_{i k h}-\Omega_{i k h} \geq \sum_{m \in M} \alpha_{k-1, m h} \theta_{i k m h}, \quad \forall i \in N \backslash\{0, n\}, \forall k \in B \backslash\{0\}, \forall h \in H$,

$$
\phi_{i} \geq \sum_{k \in B \backslash\{0\}} \sum_{h \in H} \frac{\Omega_{i k h}}{\rho_{k h}}, \quad \forall i \in N \backslash\{0, n\} .
$$

Constraints (6) and (7) define the battery level of the EB when it finishes the charging process at the PVCS located at node $i \in N \backslash\{0, n\}$ on the segment between breakpoints $k-$ 1 and $k \in B \backslash\{0\}$. Constraints (8)-(10) ensure that when the EB charges on a segment of the piecewise linear function, the energy level is between the corresponding energy levels $\alpha_{k-1, \mathrm{mh}}$ and $\alpha_{\mathrm{kmh}}$. Constraint (11) determines that the charging time at the PVCS located at node $i \in N \backslash\{0, n\}$.
$\Omega_{i k h} \leq \sum_{m \in M} \theta_{i k m h} Q_{\max }, \quad \forall i \in N \backslash\{0, n\}, \forall k \in B \backslash\{0\}, \forall h \in H$,

$\theta_{i k m h} \leq w_{m}, \quad \forall i \in N \backslash\{0, n\}, \forall k \in B \backslash\{0\}, \forall m \in M, \forall h \in H$,

$\theta_{i k m h} \leq y_{i h}+y_{n-i, h}, \quad \forall i \in N \backslash\{0, r, n\}, \forall k \in B \backslash\{0\}, \forall m \in M, \forall h \in H$,

$\theta_{r, k m h} \leq y_{r, h}, \quad \forall k \in B \backslash\{0\}, \forall m \in M, \forall h \in H$,

$\sum_{h \in H}\left(y_{i h}+y_{n-i, h}\right) \leq 1, \quad \forall i \in N_{o}$

$$
\sum_{h \in H} y_{r, h} \leq 1
$$

Constraint (12) ensures that charging is allowed only on the selected segments of the piecewise linear function. Constraint (13) determines that charging is possible only for the battery capacity selected. Constraints (14) and (15) ensure that charging is allowed only at the located PVCSs. Constraints (16) and (17) ensure that, at most, one type $h \in H$ of PVCS is located at each node.

$$
\begin{aligned}
& \delta_{i} \geq o_{i}, \quad \forall i \in N \backslash\{0, n\}, \\
& \delta_{i} \geq \phi_{i}, \quad \forall i \in N \backslash\{0, n\}, \\
& \tau_{1} \geq t_{1}, \\
& \tau_{i}=\tau_{i-1}+\delta_{i-1}+t_{i}, \quad \forall i \in N \backslash\{0,1\}, \\
& s_{i} \leq \tau_{i} \leq l_{i}, \quad \forall i \in N \backslash\{0\} .
\end{aligned}
$$

Constraints (18) and (19) define the stay time at each node, which is the maximum value between the operation time and the charging time. Constraints (20) and (21) determine the arrival time at each node. Constraints (22) ensure that the EB has to meet the TW of each node.

$$
\begin{aligned}
& z_{i j} \leq \sum_{h \in H}\left(y_{i h}+y_{n-i, h}\right), \quad \forall i \in N \backslash\{0, r, n\}, \forall j \in T \\
& z_{r, j} \leq \sum_{h \in H} y_{r, h}, \quad \forall j \in T \\
& \gamma_{i j} \leq \Delta z_{i j}, \quad \forall i \in N \backslash\{0, n\}, \forall j \in T \\
& \gamma_{i j} \leq \tau_{i}-u_{j-1}+\left(1-z_{i j}\right) u_{j}, \quad \forall i \in N \backslash\{0, n\}, \forall j \in T \backslash\{1\}
\end{aligned}
$$




$$
\sum_{j \in T} \gamma_{i j}+\left(1-\sum_{h \in H}\left(y_{i h}+y_{n-i, h}\right)\right) T_{\max } \geq \tau_{i}, \quad \forall i \in N \backslash\{0, r, n\},
$$

$$
\sum_{j \in T} \gamma_{r, j}+\left(1-\sum_{h \in H} y_{r, h}\right) T_{\max } \geq \tau_{r}
$$

$$
\lambda_{i}=g_{j} \gamma_{i j}, \quad \forall i \in N \backslash\{0, n\},
$$

$$
d_{i j} \leq \sum_{h \in H}\left(y_{i h}+y_{n-i, h}\right), \quad \forall i \in N \backslash\{0, r, n\}, \forall j \in T,
$$

$$
d_{r, j} \leq \sum_{h \in H} y_{r, h}, \quad \forall j \in T,
$$

$$
p_{i j} \leq \Delta d_{i j}, \quad \forall i \in N \backslash\{0, n\}, \forall j \in T,
$$

$$
p_{i j} \leq u_{j}-\tau_{i}+\left(1-d_{i j}\right) T_{\max }, \quad \forall i \in N \backslash\{0, n\}, \forall j \in T,
$$

$$
\% p_{i j} \leq \tau_{i}+\phi_{i}-u_{j}+\Delta+\left(1-d_{i j}\right) T_{\max }, \quad \forall i \in N \backslash\{0, n\}, \forall j \in T,
$$

$$
\sum_{j \in T} p_{i j}=\phi_{i}, \quad \forall i \in N \backslash\{0, n\}
$$

$$
\varphi_{i}=\sum_{j \in T} g_{j} p_{i j}, \quad \forall i \in N \backslash\{0, n\},
$$

$$
\psi_{i} \leq a-v, \quad \forall i \in N \backslash\{0, n\}
$$

$$
\psi_{i} \leq \lambda_{i}, \quad \forall i \in N: 0<i \leq r,
$$

$$
\% \psi_{i} \leq \lambda_{i}-\lambda_{n-i}+\psi_{n-i}-\sum_{k \in B \backslash\{0\}} \sum_{h \in H} \Omega_{n-i, k h}, \quad \forall i \in N: i>r,
$$

$$
\sum_{k \in B \backslash\{0\}} \Omega_{i k h} \leq \psi_{i}+\varphi_{i}, \quad \forall i \in N \backslash\{0, n\} .
$$

Constraints (23)-(25) ensure that there is generation only in the nodes where a PVCS was located. Constraints (26)-(28) control the time of generation to be equal to the arrival time of the EB to the PVCS. Constraint (29) calculates the energy generation until the arrival of the EB to the PVCS. This considers that the energy generated starts from the lower energy level. Constraints (30)-(32) control that the time of generation during the charging process is only for the nodes with a located PVCS. Constraints (33)-(35) estimate the time of generation to fulfill the energy requirements of the EB at its arrival. Constraint (36) calculates the energy generation during the time that the $\mathrm{EB}$ is charging.
Constraint (37) limits the available energy for charging the $\mathrm{EB}$ at each PVCS to the maximum capacity, considering the lower energy limit. Constraint (38) ensures that the available energy at the PVCS in the outbound trip is, at most, the generated energy until the arrival. Constraint (39) limits the available energy at the PVCS, in the return trip, considering the energy retrieved by the EB in the outbound trip. Constraint (40) ensures that the energy taken by the EB at each PVCS has been already generated at the end of the charging process.

$$
\begin{aligned}
& w_{m} \in\{0,1\}, \quad \forall m \in M, \\
& x_{i} \geq 0, \tau_{i} \geq 0, \quad \forall i \in N \backslash\{0\}, \\
& y_{i h} \in\{0,1\}, \quad \forall i \in N \backslash\{0, n\}, \forall h \in H, \\
& \Omega_{i k h} \geq 0, \mu_{i k h} \geq 0, \quad \forall i \in N \backslash\{0, n\}, \forall k \in B \backslash\{0\}, \forall h \in H,
\end{aligned}
$$

$\theta_{i k m h} \in\{0,1\}, \quad \forall i \in N \backslash\{n\}, \forall b \in B \backslash\{0\}, \forall m \in M, \forall h \in H$,

$$
\begin{gathered}
\phi_{i} \geq 0, \delta_{i} \geq 0, \lambda_{i} \geq 0, \varphi_{i} \geq 0, \psi_{i} \geq 0, \quad \forall i \in N \backslash\{0, n\}, \\
d_{i j} \in\{0,1\}, z_{i j} \in\{0,1\}, \quad \forall i \in N \backslash\{0, n\}, \forall j \in T, \\
\gamma_{i j} \geq 0, p_{i j} \geq 0, \quad \forall i \in N \backslash\{0, n\}, \forall j \in T .
\end{gathered}
$$

Finally, constraints (41)-(48) define the domain of the decision variables.

\section{Computational Experiments}

Aiming to test the performance of our MILP formulation for the ERCSLP, we used a set of instances based on real river transport operations, from different regions in Colombia. In these experiments, we initially focus on the capability of the MILP formulation to solve different types of instances in reasonable CPU times. Additionally, we show the features of the solution in terms of the number of opened PVCSs and the selected battery size for the EB. Also, for evaluating the economical feasibility of the solutions, we compare their objective functions, which correspond to the investment costs, against the total cost of a 10-year operation using an ICB. In some cases, the investment could have a return. Furthermore, we perform a sensitivity analysis to test the robustness of the solutions when variations occur in key features, such as the EB speed, the average radiation, and the discretization of the battery capacity.

5.1. Test Instances for the ERCSLP. Within the frame of the research project "Energética 2030," a river transport operation with EBs is planned to be implemented on the Magdalena River, in Colombia [40]. This project aims to start with a pilot model of a river transport operation using 
EBs including intermediate PVCSs. It is expected that this pilot serves as the starting point for launching future projects of electric mobility on other rivers in Colombia. Therefore, for the construction of the test instances, we consider different rivers that could have a river transport operation with EBs. For the test instances, we consider 6 of the most important rivers in Colombia: Amazonas, Caquetá, Guaviare, Magdalena, Orinoco, and Putumayo. For the Caquetá River, we build 3 instances; for the Magdalena River, we build 4 instances; for Guaviare River, we build 2 instances; and for the remaining rivers, we build one instance each. Then, the total number of instances is 12 . For each instance, we locate the set of nodes in places where there are towns or settlements. This is performed aiming to supply the excess of energy to the community and reducing the impact on the river (caused for the installation of the PVCSs). It is important to remark that most of the nodes are disconnected from the national grid because they are located in isolated areas.

For building the instances, we make the following considerations. For the discretization of the EB battery capacity, we use a delta of $1 \mathrm{kWh}$. For the energy consumption estimation, we use Crouch's formula presented by Minami and Yamachika [33]. This estimation was made for each EB possible battery capacity. We selected Crouch's formula because it is easy to implement and has the potential to produce reliable results. However, any consumption model could be used given that the energy consumption is a parameter. For estimating the weight associated to each EB possible battery capacity, we consider an energy density of $0.15 \mathrm{kWh} / \mathrm{kg}$ for a Li-ion battery, following the values presented by Epec [41]. The maximum battery capacity is bounded to a maximum weight value of a ton, which is an adequate value for the type of EB considered. For computing the travel time between two nodes, we use a constant speed of $10 \mathrm{~km} / \mathrm{h}$, which agrees with the data presented by Jaimurzina et al. [7]. It is important to remark that both the energy consumption and travel time are also affected by the direction of the river flow (i.e., with or against the river flow). For generating the TWs and service times, we consider the operation conditions of the route, for example, eating, sleeping, loading, and unloading times. For estimating the breakpoints of the piecewise linear charging function, we use the data presented by Martin et al. [42]. For determining the battery cost, we use the data of a battery pack manufacturing costs per kWh by Bloomberg New Energy Finance [43] and Battery University [44]. From this information, we set the cost as $300 \mathrm{USD} / \mathrm{kWh}$. For establishing the standalone PVCS cost, we took the information from a PVCS commercial supplier [45]. We added an additional factor to the PVCS cost for including the installation costs. For estimating the PV generation, we use the hourly averages presented by IDEAM [39]. For the total generation, we assumed a panel efficiency of $14 \%$ since this value is in the rank of commercial PV modules [46]. The lower energy level for the ESS is set at $20 \%$ of the EB battery capacity following the recommendation by Venu et al. [47].

Moreover, for evaluating how some parameters affect the structure of the solution (i.e., the number of charging stations and size of the EB's battery) and the objective function, we varied some parameters in each instance. For each of the instances, we varied the radiation, the average speed, and the delta of the discretization. These variations were performed one parameter at a time. For the radiation, we set the values to the historical maximum and minimum. For the average speed, we evaluated the set of values $6,7,8,9,11,12,13,14$, and $15 \mathrm{~km} / \mathrm{h}$. Finally, for the delta of the discretization, we evaluated the set of values $0.5,0.75,1.25$, and $1.5 \mathrm{kWh}$. After applying this variation, we had 15 additional instances for each of the original instances, for a total of 180 instances.

5.2. Experimental Environment. For solving the MILP formulation of the ERCSLP, we used the Gurobi Optimizer (version 8.1.0) with a CPU time limit of $10,800 \mathrm{~s}$ and a relative MIP optimality gap of $1 e-4$. When the optimality gap (the optimality gap is calculated as Gap = ((Dual Solution - Primal Solution)/Dual Solution)) is lesser than the relative MIP optimality gap, the solver finishes and reports the best primal bound as the optimal solution. We ran all the experiments on a computer with an Intel Xeon E5-2670 processor (with 16 cores at $2.6 \mathrm{GHz}$ ) and $64 \mathrm{~GB}$ of RAM running on Linux CentOS 6.6. The feasibility of the solutions reported by the Gurobi Optimizer was validated in an external application implemented on Java (jdk-1.8.0_162b12).

5.3. Results of the Computational Experiments. Initially, we solve the 12 original instances, and Table 1 presents the results in detail. In this table, the results are sorted based on the length of each route in a river with the shortest on top. Hereafter, we explain the information contained in each column of Table 1. Column 1 lists the instances names. Column 2 shows the total length (in $\mathrm{km}$ ) of each route. Column 3 presents the number of nodes that compose each route. Column 4 shows the number of PVCSs that were open for each instance. Column 5 presents the percentage of nodes that were opened as PVCS in each route. Column 6 contains the battery capacity (in $\mathrm{kWh}$ ) that was selected for the EB in each route. Column 7 presents the percentage of the selected battery, in terms of the maximum battery capacity. Column 8 presents the objective function value, which is the total cost. Column 9 presents the percentage for the PVCS cost, in terms of the total cost. Column 10 shows the percentage of the selected battery cost, in terms of the total cost. Finally, column 11 shows the CPU time. In Table 1 , we also included the economic comparison of the operation of the route using EB against ICB. For this comparison, we computed the total cost of the route operation using ICB during 10 years. This period was selected due to the estimated lifespan of the EB battery [48]. It is important to mention that the PVCS has a lifespan of 20 years, which is twice the lifespan of the EB battery. For comparing both operations, we needed to estimate the amount of fuel to perform each of the routes. For this estimation, we converted the total energy needed for completing each of the routes (in $\mathrm{kWh}$ ) to the amount 


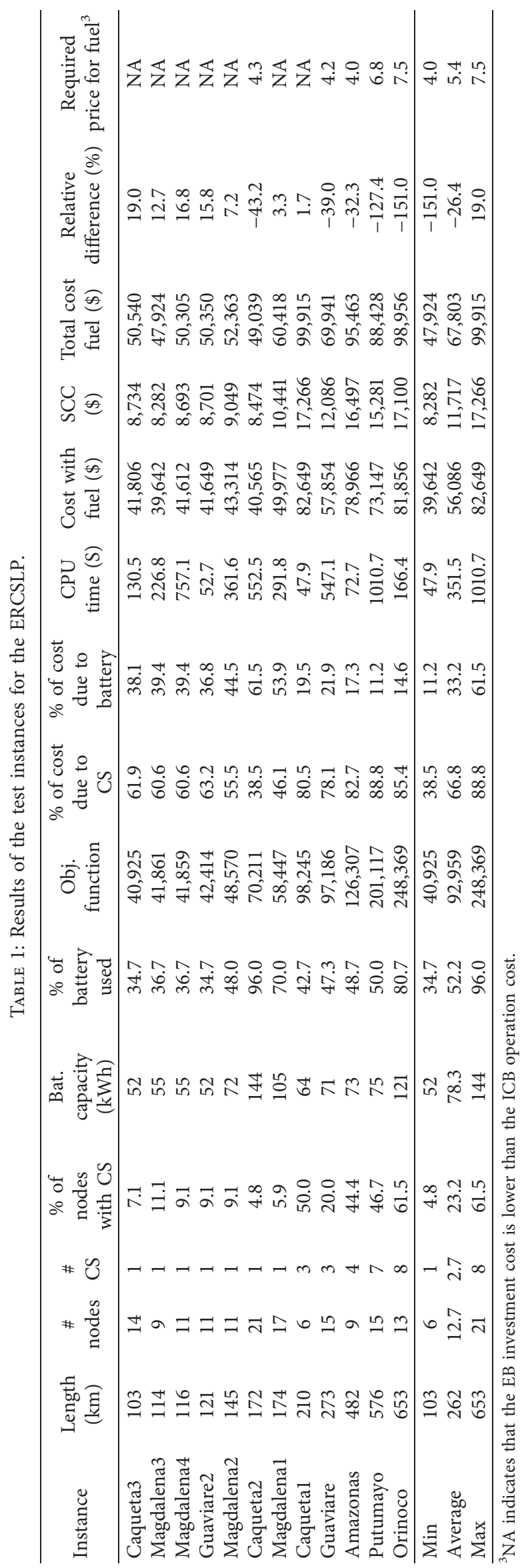


of fuel (in gallons) using the information presented by Lee [49]. For the total cost using an ICB, we considered two elements, the fuel cost and the Social Cost of Carbon (SCC). For estimating the SCC, we first computed the $\mathrm{CO}_{2}$ emission using an average value for marine Diesel [50], and then, we transformed the $\mathrm{CO}_{2}$ emission into cost considering the value presented by Nordhaus [51]. Also, for the routes where the ICB operation cost was under the investment cost of the $\mathrm{EB}$ operation, we estimated what would be the minimum fuel cost for both operations to have the same cost. From this point up, the operation with the EB will be the best economic option. From these considerations, Column 12 shows the fuel cost of performing each route using an ICB. Column 13 presents the SCC. Column 14 shows the total cost of the fuel operation (fuel cost + SCC). Column 15 shows the relative difference (Relative Difference $=$ ((Cost With Fuel - Objective Function)/Cost With Fuel)) between the investment cost of the electric mobility system and the operation cost with an ICB. Finally, Column 16 presents the required fuel price (in USD/gallon) for making both operation cost equal.

For all the instances shown in Table 1, the optimality gap of the solution was smaller than the relative MIP optimality gap, which allows us to conclude that the instances are solved optimally. The average CPU time is around 6 minutes, which is a competitive CPU time for a strategic planning problem. In average, $23.2 \%$ of the nodes are selected to open a PVCS. It is important to mention that the 4 instances with the shortest lengths (Caqueta; 3, Magdalena; 3, Magdalena; 4, and Guaviare; 2) can be operated without a PVCS if the EB has a large battery size (respecting the maximum battery size). However, in the optimal solution, one PVCS is opened in all 4 routes. These results prove that, in some cases, it is better to select a small battery size and open a PVCS. As we can expect, the longest routes (Amazonas, Orinoco, and Putumayo) require a significant number of PVCSs. From the results in Table 1, we can also see that most of the solutions required a battery size that is less than the $50 \%$ of the maximum battery size. When analyzing the distribution of the cost, in average, $66.8 \%$ of the cost is devoted to opening PVCSs. Therefore, when we consider the EB operation, the most representative cost is related to the PVCSs. When comparing the investment cost against the cost of performing the same operation with an ICB during 10 years, we notice that in 7/12 instances, the electric mobility option performs better than the ICB operation. For the remaining instances, there is a minimum of 7.5 (USD/gallon) to ensure that the electric mobility which is the best option (see Column 17). Even though this value seems very high, considering the isolations of these areas, the fuel transportation impacts the final fuel costs. Under this premises, the fuel cost could surpass the 7.5 (USD/gallon) threshold.

5.4. Sensitivity Analysis. We now provide a sensitivity analysis to show how certain modifications on some parameters can impact the objective function. To do this, we used the 192 total instances, comprised of the 12 original instances and the 180 additional instances. Firstly, we proposed a variation on the radiation level. The radiation being an input for the PVCSs, we suppose that different radiation conditions can affect the decision-making when locating the PVCSs. Secondly, considering that the average EB speed impacts the energy consumption and the travel time, we hypothesize that this variable could affect the feasibility of the solutions and the value of the objective functions. Finally, we wanted to evaluate how different deltas of the discretized battery capacity can affect the objective function value and the CPU time.

5.4.1. Solar Radiation Variation. For testing the impact of the radiation on the objective function, we evaluated three different scenarios, where we consider the months with the highest, average, and lowest solar radiation per hour. In Figure 5, we present the impact of these variations on the average value of the objective function from all the instances. This figure shows that the objective function increases when there is less solar radiation because more infrastructure is needed. However, there is not a significant variation after comparing all three scenarios. From a detailed analysis of the solutions, we found that, in most of the instances, the location and the number of PVCSs do not change in the three scenarios. The variation of the irradiation impacts the size of the battery. Considering that the PVCS cost is more expensive than the battery cost, there is not a significant variation on the average value of the objective function among the three scenarios. In Table 2, we show the solution of the instance Caquetá 1 for the three scenarios. Columns 2 to 7 present the nodes where a PVCS can be installed. The value of the cells represents the power of the selected PVCS; if a cell is empty, it is because no PVCS was installed at that associated node for that scenario. Furthermore, the last column shows the selected battery size. Table 2 also shows that the number and the location of the PVCSs are the same among the scenarios. On the other hand, the battery size changes between the scenarios with average and minimum radiation, and there is not a change between the scenarios with average and maximum radiation. In the latter, although both solutions have the same battery size the charging decisions are different. The EB must charge more energy for the average radiation scenario. Considering that variation of the radiation do not change the solutions significantly, it is adequate to use the average radiation level for this strategic problem.

5.4.2. Average Boat Speed Variation. As we mentioned before, the EB speed has a significant impact on the energy consumption. This impact has a direct effect on the number of PVCSs and EB battery capacity. Furthermore, the EB speed can also affect the solution feasibility by the TW at each node and the $T_{\max }$ for the operation. In Figure 6, we present how the variation of the EB speed affects the objective function. In this figure, we show the average value of the objective function from all the instances. It is important to note that none of the instances below $9 \mathrm{~km} / \mathrm{h}$ had a feasible solution. This figure also shows that there is a significant impact of the EB speed on the total infrastructure 


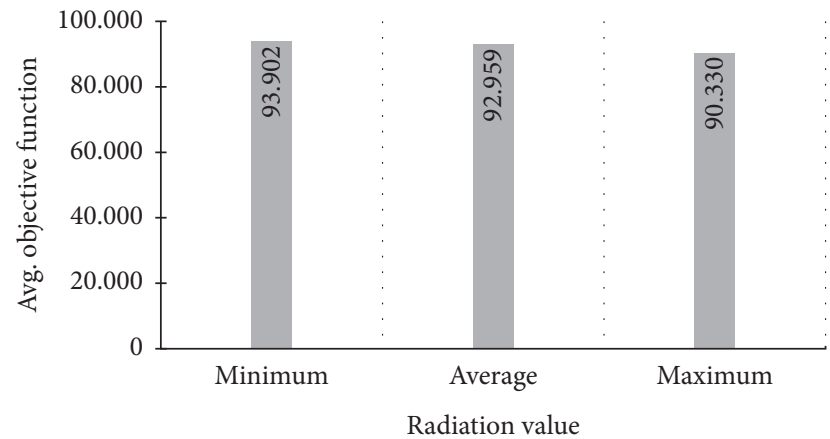

FIgURE 5: Variation of the objective function in terms of the solar radiation.

TABLe 2: Caquetá 1 instance solution for the three radiation scenarios.

\begin{tabular}{lccccccc}
\hline \multicolumn{7}{c}{ Node } \\
Scenario & 1 & 2 & 3 & 4 & 5 & 6 & Battery \\
\hline Maximum & - & $22 \mathrm{~kW}$ & $11 \mathrm{~kW}$ & - & $22 \mathrm{~kW}$ & - & $64 \mathrm{kWh}$ \\
Average & - & $22 \mathrm{~kW}$ & $11 \mathrm{~kW}$ & - & $22 \mathrm{~kW}$ & - & $64 \mathrm{kWh}$ \\
Minimum & - & $22 \mathrm{~kW}$ & $11 \mathrm{~kW}$ & - & $22 \mathrm{~kW}$ & - & $73 \mathrm{kWh}$ \\
\hline
\end{tabular}

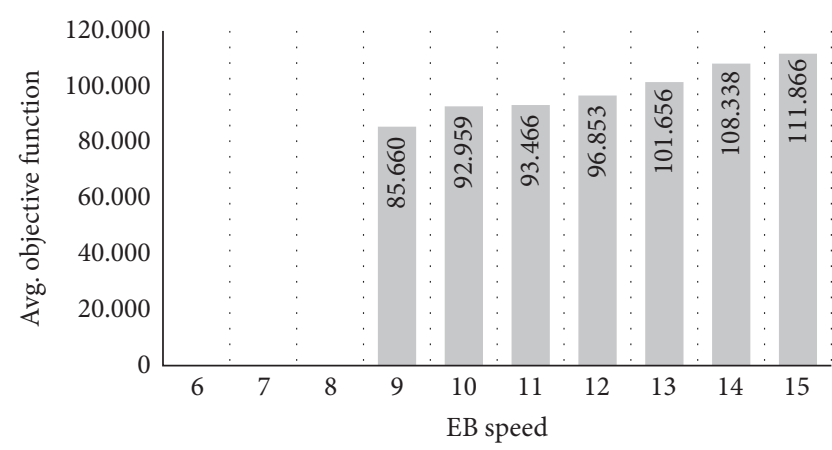

FIgURE 6: Changes in the objective function according to the average EB speed.

cost. It is important to notice that increasing the EB speed could improve the service in the operation; however, this increment on the speed will directly impact the infrastructure needed to support the operation.

5.4.3. Discretization of the Battery Capacity. As we mention in the problem description, the battery capacity is a decision variable. This variable takes discrete values from the set $M$ that represents the possible values of the battery capacity. For building that set, we define a delta value of the battery capacity, which is interpreted as the step that increases the battery capacity in the set $M$. In this analysis, we want to test how the delta of the discretization affects the objective function. For the analysis, we choose battery capacity discretization values of $0.5,0.75,1,1.25$, and $1.5 \mathrm{kWh}$ being $1 \mathrm{kWh}$ the value for the discretization of the original instances. Figure 7 shows the average results of the objective function for the different delta values. For this variation of

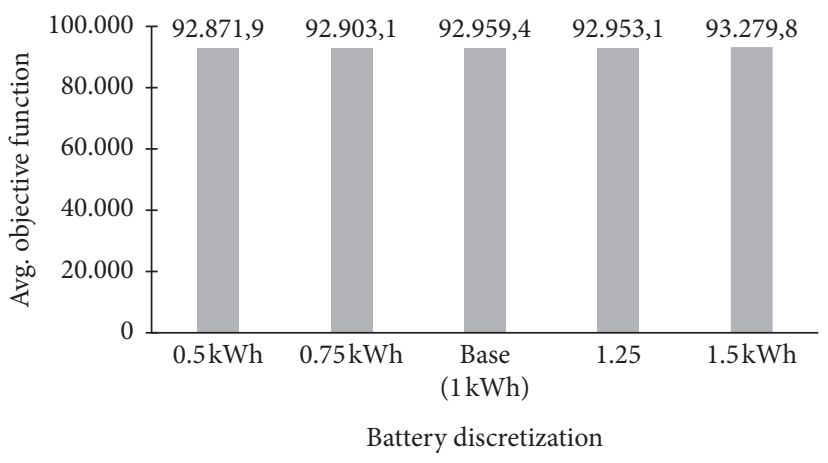

FIGURE 7: Changes in the objective function according to the battery capacity discretization.

the delta, the MILP formulation was capable of optimally solving all the instances. When we analyze the impact of this variation on the objective function, we see that, for most of the delta variations, there is not a significant impact. The only delta variation that presented an impact on the cost was $1.5 \mathrm{kWh}$. In this case, there is $0.34 \%$ increase in the objective function when compared to the results using a delta of $1 \mathrm{kWh}$. From these results, we can conclude that using a $1 \mathrm{kWh}$ delta is an acceptable choice because we can obtain competitive solutions (in terms of the objective function) and reasonable CPU times for this kind of problems.

\section{Conclusions}

In this paper, we introduced a new optimization problem named the Electric Riverboat Charging Station Location Problem (ERCSLP). This problem consists in estimating the necessary infrastructure so that an EB can perform a round trip on a river visiting several nodes. The objective of this problem is to decide the location of the PVCSs and the size of the EB battery aiming to minimize the total investment cost. This cost is defined as the sum of the PVCSs and battery costs. The ERCSLP includes some components from real river transport operations such as TW, service times, and a time limit of the route. Furthermore, this problem includes some components of the electric mobility such as the behavior of the nonlinear charging function and the variation of the solar radiation during the day. For solving this problem, we propose a MILP formulation that is able to consider all the characteristics of the proposed problem.

For testing the MILP formulation, we built a set of instances based on different river transport operations, in Colombia, including a river operation that will be a pilot for the use of an EBs. The results show that our MILP formulation is capable of optimally solving all the instances in competitive CPU times for a strategic problem. Moreover, it is important to note that there are some instances where it possible to perform the route without placing PVCSs; however, the MILP formulation shows that, in this case, it is better to place a PVCSs operating with a smaller battery. Furthermore, we found that, for most of the tested river operations, the electric mobility could be economic feasible, when compared against the same operation with internal 
combustion cost. Finally, we perform a sensitivity analysis evaluating the impact on the objective function when we vary some parameters. We determinate that the EBs speed has a significant impact on the objective function; conversely, the variation of solar radiation had a negligible impact.

\section{Nomenclature}

$N: \quad$ Set of nodes

0 : $\quad$ Starting point

$n$ : $\quad$ Final destination

$r$ : $\quad$ Return node

$N_{0}$ : Nodes to visit on the outbound trip

$N_{r}$ : Nodes to visit on the return trip

$o_{i}: \quad$ Service time at node $i \in N \backslash\{0, n\}$

$s_{i}$ : $\quad$ Earliest possible arrival time at node $i \in N \backslash\{0\}$

$l_{i}: \quad$ Latest possible arrival time at node $i \in N \backslash\{0\}$.

$t_{i}: \quad$ Travel time between nodes $i-1$ and $i \in N \backslash\{0\}$

$T_{\max }$ : Time limit

$M: \quad$ Set of battery capacities

$c_{m}: \quad$ Acquisition cost of battery capacity $m \in M$

$e_{i m}$ : Energy consumption between nodes $i-1$ and $i \in N \backslash\{0\}$ using a EB with a battery capacity of $m \in M$

$H$ : $\quad$ Set of different types of PVCSs' technologies

$f_{i h}$ : Cost of installing a PVCS at node $i \in N \backslash\{0, n\}$ of type $h \in H$

$B$ : $\quad$ Set of breakpoints of the piecewise linear approximation

$\rho_{k h}$ : Slope of the segment defined between breakpoints $k-1$ and $k \in B \backslash\{0\}$ charging rate $h \in H$

$\alpha_{k, m h}$ : Upper bound of the battery level of the segment defined between breakpoints $k-1$ and $k \in B \backslash\{0\}$ with charging rate $h \in H$ for a battery capacity $m \in M$

$T: \quad$ Set of time intervals

$\Delta: \quad$ Length of the time intervals

$u_{j}$ : Upper bound (in hours) of interval $j \in T$

$g_{j}$ : Power generation of interval $j \in T$

a: Capacity of the ESS

$v$ : Lower energy limit of the ESS

$w_{m}$ : Binary variable equal to 1 if the EB battery capacity takes a value of $m \in M$, and 0 otherwise

$y_{i h}$ : Binary variable equal to 1 if a PVCS of type $h \in H$ is opened at node $i \in N \backslash\{0, n\}$, and 0 otherwise

$x_{i}$ : $\quad$ Battery level when the EB arrives at node $i \in N \backslash\{0\}$

$\tau_{i}$ : Arrival time of the EB at node $i \in N \backslash\{0\}$

$\Omega_{i k h}$ : Charged energy when the EB charges at the PVCS of type $h \in H$ located at node $i \in N \backslash\{0, n\}$ between breakpoints $k-1$ and $k \in B \backslash\{0\}$

$\mu_{i k h}$ : Battery level when the EB charges at the PVCS of type $h \in H$ located at node $i \in N \backslash\{0, n\}$ between breakpoints $k-1$ and $k \in B \backslash\{0\}$

$\theta_{i k m h}$ : Binary variable equal to 1 if the EB charges on the segment between breakpoint $k-1$ and $k \in B \backslash\{0\}$ using a battery capacity of $m \in M$ at the node $i \in N \backslash\{0, n\}$, and 0 otherwise

$\phi_{i}$ : $\quad$ Charging time at the PVCS located at node $i \in N \backslash\{0, n\}$ $\delta_{i}: \quad$ Stay time at the PVCS located at node $i \in N \backslash\{0, n\}$

$\gamma_{i j}$ : Fraction of the time interval $j \in T$ that is being used to generate energy before the arrival of $\mathrm{EB}$ at the PVCS located at node $i \in N \backslash\{0, n\}$

$p_{i j}$ : Fraction of the time interval $j \in T$ that is being used to generate energy during the charging process of EB at the PVCS located at node $i \in N \backslash\{0, n\}$

$z_{i j}$ : $\quad$ Binary variable equal to 1 if the PVCS located at node $i \in N \backslash\{0, n\}$ is generating energy before the arrival of the $\mathrm{EB}$ at the time interval $j \in T$

$d_{i j}$ : Binary variable equal to 1 if the PVCS located at node $i \in N \backslash\{0, n\}$ is generating energy during the charging process of the EB at the time interval $j \in T$

$\lambda_{i}$ : Total energy generated by the PVCS located at node $i \in N \backslash\{0, n\}$ before the arrival time of the EB

$\psi_{i}$ : Available energy at the PVCS located at node $i \in N \backslash\{0, n\}$ before the arrival time of the EB

$\varphi_{i}$ : Energy generated while the EB is charging at the PVCS located at node $i \in N \backslash\{0, n\}$.

\section{Data Availability}

The data used to support the findings of this study are available from the corresponding author upon request.

\section{Conflicts of Interest}

The authors declare that there are no conflicts of interest regarding the publication of this paper.

\section{Acknowledgments}

The authors would like to thank Universidad EAFIT for supporting this research through the Research Assistantship grant from project 828-000068. This research has also been developed in the framework of the "ENERGETICA 2030" Research Program, with code 58667 in the "Scientific Colombia" initiative, funded by The World Bank through the call "778-2017 Scientific Ecosystems," managed by the Colombian Administrative Department of Science, Technology, and Innovation (COLCIENCIAS). The authors would like to thank Jorge Lozano for his assistance in the instances building automation and his contribution in the literature review and Simón Polanía for his help in the construction of the images. Additionally, the authors would like to thank Universidad EAFIT scientific computing center (APOLO) for its support for the computational experiments.

\section{References}

[1] D. Newman, P. Wells, C. Donovan, P. Nieuwenhuis, and H. Davies, "Urban, sub-urban or rural: where is the best place for electric vehicles?" International Journal of Automotive Technology and Management, vol. 14, no. 3/4, pp. 306-323, 2014.

[2] W. J. Requia, M. Mohamed, C. D. Higgins, A. Arain, and M. Ferguson, "How clean are electric vehicles? evidence-based review of the effects of electric mobility on air pollutants, 
greenhouse gas emissions and human health," Atmospheric Environment, vol. 185, pp. 64-77, 2018.

[3] J. W. Choi and D. Aurbach, "Promise and reality of postlithium-ion batteries with high energy densities," Nature Reviews Materials, vol. 1, no. 4, 2016.

[4] R. H. Ashique, Z. Salam, M. J. Bin Abdul Aziz, and A. Rauf Bhatti, "Integrated photovoltaic-grid dc fast charging system for electric vehicle: a review of the architecture and control," Renewable and Sustainable Energy Reviews, vol. 69, pp. 1243-1257, 2017.

[5] K. Branker, M. J. M. Pathak, and J. M. Pearce, "A review of solar photovoltaic levelized cost of electricity," Renewable and Sustainable Energy Reviews, vol. 15, no. 9, pp. 4470-4482, 2011.

[6] P. Bara Neto, R. J. Sánchez, and G. Wilmsmeier, “Amazonia: hacia un desarrollo sustentable e integrado," 2007.

[7] A. Jaimurzina, W. Gordon, and D. Montiel, "Eficiencia energética y movilidad eléctrica fluvial: soluciones sostenibles para la amazonía," 2017.

[8] M. Grooten and R. Almond, "Informe planeta vivo-2018," Technical report, WWF, Gland, Switzerland, 2018.

[9] L. A. Ponce, Estudio del potencial de las embarcaciones solares en la Amazonía: Caso de estudio Río Napo, Ph.D. thesis, ETSI_ Diseno, Madrid, Spain, 2017.

[10] A. Miniguano and P. Elizabeth, "Design of a business plan intended for the creation of the fluvial transport company Kara solar in Pastaza Province with positive social and environmental impact," B.S. thesis, PUCE, Quito, Ecuador, 2019.

[11] Patrimonio Natural, CEPAL, Parques Nacionales Naturales, and Gordon and Betty Moore Foundation, Amazonia Posible y Sostenible, 2013.

[12] F. Baouche, R. Billot, R. Trigui, and N.-E. El Faouzi, "Efficient allocation of electric vehicles charging stations: optimization model and application to a dense urban network," IEEE Intelligent Transportation Systems Magazine, vol. 6, no. 3, pp. 33-43, 2014.

[13] J. Lin, W. Zhou, and O. Wolfson, "Electric vehicle routing problem," Transportation Research Procedia, vol. 12, pp. 508-521, 2016.

[14] S. Pourazarm, C. G. Cassandras, and A. Malikopoulos, "Optimal routing of electric vehicles in networks with charging nodes: a dynamic programming approach," in Proceedings of the 2014 IEEE International Electric Vehicle Conference (IEVC), pp. 1-7, Florence, Italy, December 2014.

[15] S. Cui, H. Zhao, H. Wen, and C. Zhang, "Locating multiple size and multiple type of charging station for battery electricity vehicles," Sustainability, vol. 10, no. 9, p. 3267, 2018.

[16] Z.-H. Zhu, Z.-Y. Gao, J.-F. Zheng, and H.-M. Du, "Charging station location problem of plug-in electric vehicles," Journal of Transport Geography, vol. 52, pp. 11-22, 2016.

[17] Z. Sun, W. Gao, B. Li, and L. Wang, "Locating charging stations for electric vehicles," Transport Policy, 2018.

[18] X. Xi, R. Sioshansi, and V. Marano, "Simulation-optimization model for location of a public electric vehicle charging infrastructure," Transportation Research Part D: Transport and Environment, vol. 22, pp. 60-69, 2013.

[19] S. Mehar and S. Mohammed Senouci, "An optimization location scheme for electric charging stations,"vol. 1, pp. 1-5, in Proceedings of the 2013 International Conference on Smart Communications in Network Technologies (SACONET), vol. 1, pp. 1-5, IEEE, Paris, France, June 2013.

[20] J. He, H. Yang, T.-Q. Tang, and H.-J. Huang, "An optimal charging station location model with the consideration of electric vehicle's driving range," Transportation Research Part C: Emerging Technologies, vol. 86, pp. 641-654, 2018.

[21] M. H. Moradi, M. Abedini, S. M. R. Tousi, and S. M. Hosseinian, "Optimal siting and sizing of renewable energy sources and charging stations simultaneously based on differential evolution algorithm," International Journal of Electrical Power \& Energy Systems, vol. 73, pp. 1015-1024, 2015.

[22] M. R. Mozafar, M. H. Moradi, and M. H. Amini, "A simultaneous approach for optimal allocation of renewable energy sources and electric vehicle charging stations in smart grids based on improved GA-PSO algorithm," Sustainable Cities and Society, vol. 32, pp. 627-637, 2017.

[23] M. H. Amini, M. P. Moghaddam, and O. Karabasoglu, "Simultaneous allocation of electric vehicles' parking lots and distributed renewable resources in smart power distribution networks," Sustainable Cities and Society, vol. 28, pp. 332-342, 2017.

[24] J. Yang and H. Sun, "Battery swap station location-routing problem with capacitated electric vehicles," Computers \& Operations Research, vol. 55, pp. 217-232, 2015.

[25] W. Li-ying and S. Yuan-bin, "Multiple charging station location-routing problem with time window of electric vehicle," Journal of Engineering Science \& Technology Review, vol. 8, no. 5, 2015.

[26] M. Schiffer and G. Walther, "The electric location routing problem with time windows and partial recharging," European Journal of Operational Research, vol. 260, no. 3, pp. 995-1013, 2017.

[27] M. Schiffer and G. Walther, "Strategic planning of electric logistics fleet networks: a robust location-routing approach," Omega, vol. 80, pp. 31-42, 2018.

[28] S. Cui, H. Zhao, and C. Zhang, "Multiple types of plug-in charging facilities' location-routing problem with time windows for mobile charging vehicles," Sustainability, vol. 10, no. 8, p. 2855, 2018.

[29] A. Montoya, C. Guéret, J. E. Mendoza, and J. G. Villegas, "The electric vehicle routing problem with nonlinear charging function," Transportation Research Part B: Methodological, vol. 103, pp. 87-110, 2017.

[30] D. Villa, A. Montoya, and J. M. Ciro, "The electric boat charging problem," Production, vol. 29, 2019.

[31] W. Zhang, X. Yan, and D. Zhang, "Charging station location optimization of electric ship based on backup coverage model," TransNav, the International Journal on Marine Navigation and Safety of Sea Transportation, vol. 11, no. 2, p. 137, 2017.

[32] D. Villa and A. Montoya, "A taxonomy of energy consumption models for electric vehicles," in Proceedings of the MOVICI-MOYCOT 2018: Joint Conference for Urban Mobility in the Smart City, Medellin, Colombia, April 2018.

[33] S. Minami and N. Yamachika, "A practical theory of the performance of low velocity boat," Journal of Asian Electric Vehicles, vol. 2, no. 1, pp. 535-539, 2004.

[34] H. Schwanecke, "On the propulsion quality of ships operating at restricted water depth," Ocean Engineering, vol. 6, no. 6, pp. 571-580, 1979.

[35] N. Thi Ngoc Hoa, B. Ngoc Vu, N. Tu Tran, N. Manh Chien, and T. Hien Le, "Numerical investigating the effect of water depth on ship resistance using rans CFD method," 2019.

[36] S. Pelletier, O. Jabali, G. Laporte, and M. Veneroni, "Battery degradation and behaviour for electric vehicles: review and numerical analyses of several models," Transportation Research Part B: Methodological, vol. 103, pp. 158-187, 2017. 
[37] H. S. Rauschenbach, Solar Cell Array Design Handbook: The Principles and Technology of Photovoltaic Energy Conversion, Springer Science \& Business Media, Berlin, Germany, 2012.

[38] A. Rauf Bhatti, Z. Salam, M. J. Bin Abdul Aziz, K. P. Yee, and R. H. Ashique, "Electric vehicles charging using photovoltaic: status and technological review," Renewable and Sustainable Energy Reviews, vol. 54, pp. 34-47, 2016.

[39] IDEAM, Atlas de Radiación Solar, Ultravioleta y Ozono de Colombia, IDEAM, Bogotá, Colombia, 2019, http://atlas. ideam.gov.co/visorAtlasRadiacion.html.

[40] Icetex Energética 2030, Colombia Científica, 2017, http:// colombiacientifica.gov.co/colombia/.

[41] Epec, "Battery cell comparison," 2019, https://www.epectec. com/batteries/cell-comparison.html.

[42] U. Martin, L. Weiß, M. Suriyah, and T. Leibfried, "E-mobility in car parks-guidelines for charging infrastructure expansion planning and operation based on stochastic simulations," in Proceedings of the EVS28 International Electric Vehicle Symposium and Exhibition, pp. 1-12, Goyang, South Korea, May 2015.

[43] Bloomberg New Energy Finance, Electric Cars to Reach Price Parity by 2025, 2017, https://about.bnef.com/blog/electriccars-reach-price-parity-2025/.

[44] Battery University, Cost of Mobile and Renewable Power, Battery University, Richmond, Canada, 2019, https:// batteryuniversity.com/learn/article/bu_1006_cost_of_mobile_ power.

[45] Autosolar, Kit solar aislada, 2019, https://autosolar.es/kitsolar-aislada.

[46] P. Reinhard, S. Buecheler, and T. An, "Technological Status of $\mathrm{Cu}$ (In, ga)(se, s) 2-based photovoltaics," Solar Energy Materials and Solar Cells, vol. 119, pp. 287-290, 2013.

[47] C. Venu, Y. Riffonneau, S. Bacha, and Y. Baghzouz, "Battery storage system sizing in distribution feeders with distributed photovoltaic systems," in Proceedings of the 2009 IEEE Bucharest PowerTech, pp. 1-5, IEEE, Bucharest, Romania, June 2009.

[48] Energy Sage, How to Choose the Best Battery for a Solar Energy System, Energy Sage, Boston, MA, USA, 2019, https://www. energysage.com/solar/solar-energy-storage/what-are-the-bestbatteries-for-solar-panels/.

[49] E.-C. Lee, "On the water: save fuel, money: running your boat by the numbers," 2013, https://ncseagrant.ncsu.edu/coastwatch/ previous-issues/2013-2/summer-2013/on-the-water-save-fuelmoney-running-your-boat-by-the-numbers/.

[50] Sailors for the Sea, Carbon Footprint, 2019, http://www. sailorsforthesea.org/programs/green-boating-guide/carbonfootprint.

[51] W. D. Nordhaus, "Revisiting the social cost of carbon," Proceedings of the National Academy of Sciences, vol. 114, no. 7, pp. 1518-1523, 2017. 